\title{
Résultats d'une analyse du milieu visant à déterminer le niveau d'erreur de réfraction non corrigée chez les enfants des écoles primaires des Premières nations de l'Ontario
}

\author{
A. Paul Chris, DO, \\ Directeur général, \\ Vision Institute of Canada \\ Nancy L. Young, PhD, \\ Professeure et titulaire \\ de la chaire de recherche, \\ Université Laurentienne \\ Kayla Belanger, BPHE, \\ étudiante-chercheuse, \\ Université Laurentienne \\ Lyndsay Greasley, BSc, \\ étudiante-chercheuse, \\ Université Laurentienne
}

\begin{abstract}
RÉSUMÉ
Un sondage a été élaboré et utilisé pour déterminer le niveau et la qualité des services de soins de la vue offerts aux enfants des écoles primaires des Premières nations de l'Ontario et déterminer indirectement le niveau d'erreurs de réfraction non corrigées chez les enfants des Premières nations. Dans l'ensemble, les résultats du sondage ont révélé que 1 enfant sur 4 portait des lunettes. Les résultats du sondage indiquent que les collectivités éloignées qui reçoivent la visite d'un optométriste sont plus susceptibles d'avoir moins de cas d'erreurs de réfraction non corrigées que les collectivités non éloignées. Les résultats portent à croire que les examens oculaires complets effectués par des optométristes en visite dans la collectivité seraient le moyen le plus efficace d'améliorer la vision et l'état de santé oculaire des enfants des Premières nations.
\end{abstract}

elon l'Enquête nationale auprès des ménages de 2011 de Statistique Canada, un peu plus de 300000 Autochtones résident en Ontario, ce qui représente près de $22 \%$ de la population autochtone totale du Canada et $2 \%$ de la population totale de l'Ontario. De plus, il y a un peu plus de 200000 membres des Premières nations en Ontario et ils représentent donc la majorité de la population autochtone de la province et près de $25 \%$ de tous les membres des Premières nations au Canada. Parmi les membres des Premières nations ayant un statut d'Indien inscrit, 37 \% vivent dans une réserve ${ }^{1}$. Soixante pour cent (60\%) des enfants des Premières Nations vivant dans une réserve vivent dans la pauvreté, ce qui représente plus du triple du taux de pauvreté infantile au Canada, qui est de $18 \%{ }^{2}$.

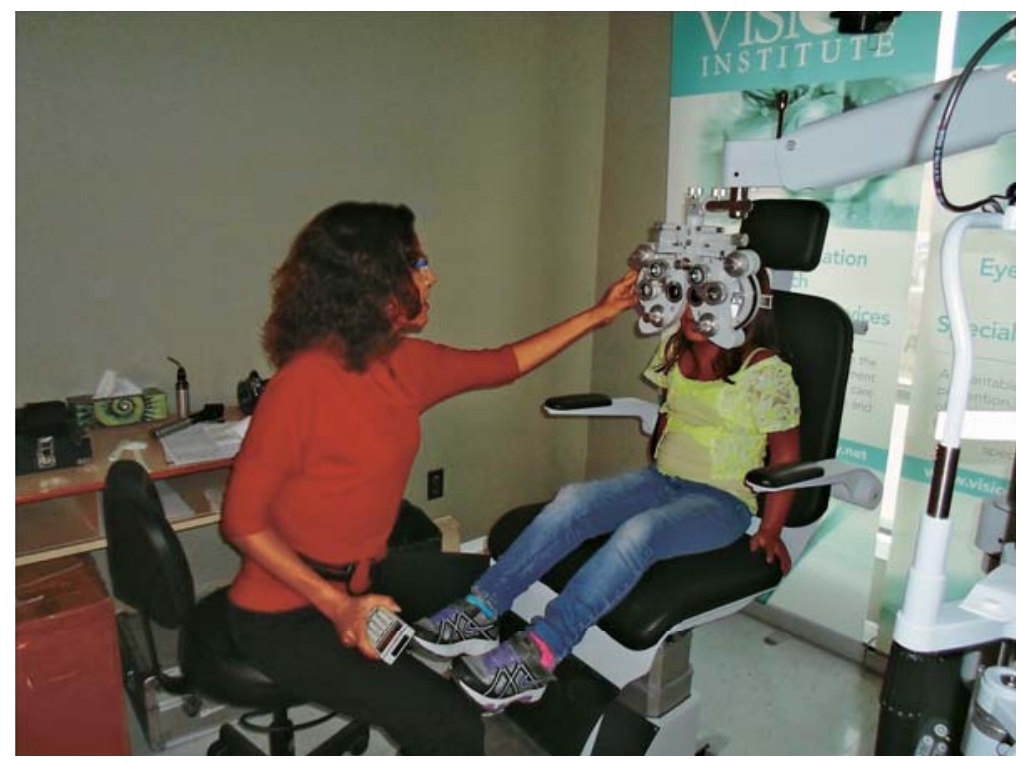

Dr Dana Blakolmer examinant un jeune étudiant à l'école primaire de la Première Nation des M'Chigeeng. 
Il existe une disparité si importante en matière de santé entre les peuples autochtones et la population globale au Canada qu'elle peut être considérée comme une épidémie nationale 3 . Il est bien établi que les Premières nations, les Métis et les Inuits connaissent des taux disproportionnés de maladies chroniques, de suicide, d'abus, de toxicomanie et de problèmes de santé mentale en comparaison de ceux qui sont observés dans la population générale ${ }^{4,5}$. Cela est souvent associé aux déterminants sociaux de la santé - tels que reconnus par l'Organisation mondiale de la Santé - notamment un statut socioéconomique plus faible, moins de possibilités d'emplois et d'éducation et un accès réduit aux biens et aux services de base tels que des aliments nutritifs et de l'eau propre ${ }^{5,6}$. De telles inégalités, conjuguées aux effets intergénérationnels du colonialisme, grâce à la Loi sur les Indiens de 1876 et au système des pensionnats, ont pour conséquence de créer de moins bons résultats en matière de santét.

Les troubles de la vue et les problèmes de santé oculaire sont un de ces résultats. Plusieurs études ont rapporté une prévalence plus élevée de l'astigmatisme chez les enfants autochtones américains et les enfants des Premières nations, en plus de problèmes d'acuité visuelle et de manque d'adhérence au port de lunettes plus importants ${ }^{7,8}$. Les cas d'erreurs de réfraction non corrigées, définis pour les besoins de cette étude comme le pourcentage d'enfants qui ont besoin de lunettes, mais n'en ont pas ou ne les portent pas, sont un marqueur clé de la santé oculaire dans une collectivité. L'erreur de réfraction est importante parce qu'elle peut conduire à une déficience visuelle à long terme si elle n'est pas diagnostiquée et traitée rapidement ${ }^{8}$, ce qui a pour résultat de perturber l'apprentissage chez les enfants et de compromettre la réussite scolaire.

Un projet de recherche pilote mené par l'auteur principal a permis de constater un taux très élevé d'erreurs de réfraction non corrigées dans une étude menée auprès des élèves des écoles primaires (de la prématernelle à la $8 \mathrm{e}$ année) dans deux écoles primaires des Premières nations de Sagamok et M'Chigeeng. Dans cette étude de deux écoles, 250 enfants ont subi des examens oculaires complets, ce qui a permis de calculer que l'incidence des erreurs de réfraction non corrigée était de $86 \%$. Seulement 4,4\% de tous les enfants examinés (11 sur 250) portaient des lunettes au moment de leur examen (voir l'annexe 1). Des études ont révélé qu'environ $25 \%$ des enfants de la maternelle à la $8 \mathrm{e}$ année pourraient devoir porter des lunettes ${ }^{8}$. Comme les erreurs de réfraction non corrigées sont une cause évitable de déficience visuelle, l'élimination des risques évitables de cécité est une priorité dans l'initiative Vision 2020 de l'Organisation mondiale de la Santé.

Malgré cette évidente disparité en matière de santé, il n’y a pas d'études récentes sur la santé oculaire et la prévalence des troubles de la vision chez les Autochtones dans la littérature scientifique, en particulier les publications portant sur les enfants des Premières Nations. Cependant, il est essentiel de diagnostiquer et de corriger les problèmes de vision à un stade précoce chez les enfants, puisque l'intervention précoce réduit de façon significative le risque d'amblyopie et de perte de vision ${ }^{9-12}$. De plus, étant donné que les enfants autochtones sont décrits comme des apprenants visuels ${ }^{13}$, et qu'une part importante de l'apprentissage de l'enfant est fondé sur la vision, l'erreur de réfraction non corrigée a des répercussions importantes sur la lecture, l'écriture et l'expérience éducative globale9. Beaucoup d'enfants pensent que leur mauvaise vue est normale et les parents, les soignants et les enseignants peuvent ne pas connaître les signes souvent subtils de la déficience visuelle, comme les maux de tête ou la fatigue oculaire, une capacité d'attention de courte durée ou le fait de perdre sa ligne lors de la lecture. Ainsi, l'accès tardif ou insuffisant aux soins de la vue pose un obstacle important à l'alphabétisation, à l'éducation et au développement social ${ }^{8}$.

Cet écart reflète un besoin non satisfait et justifie l'évaluation de l'état actuel de la santé oculaire et des services de soins de la vue fournis aux enfants des Premières nations de l'Ontario, notamment leur accessibilité et l'utilisation d'examens de la vue complets en temps opportun. Toutefois, cela exige une exploration critique du profil culturel, économique et géographique unique de chaque Première nation, ainsi que des barrières qui en résultent, que ce soit au niveau individuel ou collectif.

Diverses études internationales ont montré que les enfants issus de familles à faible revenu et à faible niveau de scolarité sont plus susceptibles de souffrir de déficiences visuelles et moins susceptibles d'être diagnostiqués et traités pour des problèmes de vision ${ }^{14-16}$. Étant donné qu'une plus grande proportion d'enfants autochtones proviennent de milieux défavorisés par rapport à l'ensemble de la population, ils présentent donc un risque plus élevé de troubles de la vue non traités ${ }^{14}$. Un manque d'accès aux soins a également été signalé comme étant un facteur important qui affecte la santé de la vision chez les enfants et était précédemment considéré comme une des premières causes de l'erreur de réfraction non corrigée ${ }^{15-17}$. Il faut également tenir compte de la vaste géographie du Canada; beaucoup de membres des Premières nations vivent dans des collectivités rurales 
et nordiques où l'optométriste le plus proche se trouve souvent à des dizaines, voire à des centaines de kilomètres. Si l'on ajoute à cela l'absence de compensation pour les frais de déplacement (ou une compensation insuffisante), le problème d'accès à un véhicule et les conditions de voyage souvent dangereuses, les défis sont magnifiés. Ces réalités sont souvent sous-estimées.

Le dépistage des troubles de la vue par les agents de santé communautaires est une solution qui a été envisagée. Une récente étude menée en 2015 à la Première Nation de Lac Seul par des chercheurs de l'Hôpital pour enfants malades et de l’Université McMaster a démontré que même un programme de dépistage des troubles de la vue bien conçu ne convenait pas aux enfants qui fréquentent l'école dans une collectivité éloignée des Premières nations et que des examens de la vue complets doivent être la norme d'excellence des soins (voir l'annexe 2). Un examen de la vue complet comprend une évaluation des antécédents oculaires et médicaux, de l'acuité visuelle, de la coordination des yeux, de la réfraction et de la santé oculaire. Cette évaluation approfondie devrait assurer l’identification de tout problème oculaire.

La meilleure solution consiste à établir des partenariats entre les optométristes et les Premières nations, ce qui, selon nous, devrait être la norme de référence en raison de leur situation géographique unique. Cependant, compte tenu des ressources humaines et du financement gouvernemental limités, il est impératif de fournir des données probantes à l'appui de l'attribution d'optométristes aux collectivités. Pour répondre aux besoins de la population de la façon la plus efficace, nous devons d'abord déterminer quelles sont les collectivités où les besoins sont les plus pressants et ensuite allouer les ressources en conséquence.

Cette étude visait (A) à faire une estimation du besoin de services basée sur les niveaux d'erreur de réfraction corrigée en comparaison avec les normes attendues, et (B) à déterminer le niveau et la fréquence des services de soins de la vue offerts aux enfants de la prématernelle à la 8 e année qui fréquentent des écoles élémentaires des Premières nations administrées par la bande en Ontario. Tous ces renseignements peuvent guider l'attribution de services aux Premières nations.

\section{MÉTHODOLOGIE}

\section{Approbation du comité d'éthique}

Le protocole de l'étude a été examiné par les représentants de l'Organisation des Chefs de l'Ontario et de l'Institut de la vision du Canada. Il a également été examiné et approuvé par le Comité d'éthique de la recherche de l’Université Laurentienne le 9 octobre 2015.

\section{Participants}

Un sondage a été élaboré et mis en œuvre en collaboration avec les Chiefs of Ontario, le Vision Institute of Canada et l'Université Laurentienne. Le questionnaire du sondage comprenait 13 questions portant sur les services de soins oculaires offerts aux enfants des Premières nations de l'Ontario qui fréquentaient des écoles primaires administrées par la bande. Chacune des 77 écoles primaires des Premières nations de l'Ontario (de la maternelle à la 8e année) a été invitée à y participer, ce qui représente environ 9500 enfants. Les écoles qui offraient également les études secondaires n’ont pas inclut les données démographiques pour les élèves de la 9e à la 12e année.

\section{Procédure}

Les Chiefs of Ontario ont envoyé la page d'information de l'étude (voir l'annexe 3) et le questionnaire du sondage (voir l'annexe 4) aux représentants des conseils scolaires de chaque école administrée par la bande par courrier électronique le 26 octobre 2015. L'appui des représentants des conseils scolaires était nécessaire pour envoyer le lien vers le questionnaire en ligne du sondage au directeur de l'école. Cette mesure était nécessaire étant donné l'incohérence des renseignements sur les courriels des personnes-ressources dans la base de données des Chiefs of Ontario.

Les participants disposaient de deux options pour remplir le sondage. La première consistait à imprimer le questionnaire du sondage joint au courriel et à le remplir à la main, puis à l'envoyer par télécopieur au numéro du Vision Institute of Canada qui figurait sur la page d'information de l'étude. Il était aussi possible de remplir une version en ligne via REDCap, une base de données en ligne sécurisée accessible par un lien sur la page d'information ${ }^{18}$. Comme expliqué dans la page d'information, la soumission du questionnaire rempli était considérée comme un consentement implicite.

Le sondage exigeait qu'un représentant de chaque école, comme le représentant du conseil scolaire, le directeur de l'école, l'enseignant ou l'infirmière, se rende dans chaque salle de classe et compte le nombre d'enfants qui portaient des lunettes et le nombre d'enfants qui ne portaient pas de lunettes le jour du compte (les nombres comptés n'étaient pas 
nécessairement représentatifs du nombre réel d'élèves). Le reste du questionnaire pouvait être rempli indépendamment de la collecte de données et portait sur l'information concernant le type et la fréquence des services de soins de la vue fournis à la collectivité et le rôle de l'école dans l'identification des enfants ayant besoin d'une évaluation de la vue.

Suite à la distribution du sondage, deux assistantes étudiantes de l'Université Laurentienne ont fait un suivi et téléphoné à chaque directeur d'école pour s'assurer que le représentant du conseil scolaire de l'école leur avait fait parvenir les documents du sondage. Au cours de ces appels téléphoniques de suivi, certains directeurs d'école ont fourni une adresse électronique pour que le questionnaire leur soit envoyé directement, car ils n'avaient pas encore reçu une copie de leur représentant du conseil scolaire. S'il n'avait pas été possible de communiquer avec le directeur d'école après au moins 3 tentatives avant le 22 décembre 2015, l'école était placée sur une liste d'envoi pour que le questionnaire soit envoyé directement à l'école par le Vision Institute of Canada. Cette étape a été entreprise pour s'assurer que chaque école avait eu la possibilité de participer en raison du nombre d'écoles qui ont signalé que leur représentant du conseil scolaire ne leur avait pas fait parvenir le questionnaire du sondage.

\section{Analyse des données}

La collecte des données a officiellement pris fin le 12 février 2016, après quoi le sondage a été mis hors ligne. Le pourcentage d'enfants qui portaient des lunettes a été obtenu en divisant le nombre d'enfants qui portaient des lunettes par le nombre total d'enfants dans l'école le jour où le dénombrement a été effectué (c'est-à-dire la somme du nombre d'enfants portant des lunettes et n'en portant pas). En fonction des présences lors de cette journée précise, ce nombre peut être égal ou non au total de l'effectif scolaire. Des études antérieures ont révélé qu'environ $25 \%$ des enfants de la prématernelle à la 8 e année sont susceptibles de porter des lunettes ${ }^{8}$. En comparant le pourcentage rapporté de chaque école avec cette valeur attendue, nous pouvons déterminer, de façon très élémentaire, les besoins en matière de soins en optométrie dans la collectivité.

En plus du groupe global, les données étaient également organisées en quatre catégories principales : si l'école avait un optométriste visiteur, si l'école avait une autre forme d'évaluation de la vision (par exemple, le dépistage dans les réserves par une infirmière praticienne, une infirmière d'école, un enseignant, etc.), si les élèves ont vu un optométriste à l'extérieur de la réserve et si l'école était située dans une collectivité isolée.

\section{Présentation des données}

Un rapport d'une page qui résume les conclusions de cette étude a été donné à toutes les écoles primaires administrées par la bande en Ontario. Les rapports, propres à chaque collectivité, indiquaient où se trouvaient les optométristes les plus proches, le taux d'erreur de réfraction non corrigée à l'école et les résultats individuels de l'école en comparaison avec les autres écoles qui avaient participé à l'étude et les normes prévues. Deux types de rapports ont été employés, l'un dans un format écrit et l'autre avec des infographies et des éléments visuels supplémentaires pour rendre les données disponibles d'une manière culturellement appropriée.

\section{RÉSULTATS}

\section{Description des répondants}

Au total, 33 des 77 écoles primaires des Premières nations (43\%) ont répondu au sondage, 19 par télécopieur et 14 en ligne via REDCap (figure et tableau 1).

Tableau 1 : Taux de réponse des collectivités é loignées et des collectivités non éloignées

\begin{tabular}{|l|c|c|c|}
\hline & $\begin{array}{l}\text { Nombre de } \\
\text { collectivités }\end{array}$ & $\begin{array}{l}\text { Nombre de } \\
\text { répondants }\end{array}$ & $\begin{array}{l}\text { Taux de } \\
\text { réponse }\end{array}$ \\
\hline Éloignée Oui & 32 & 12 & $\begin{array}{l}37,5 \% \\
46,7 \%\end{array}$ \\
\hline Non & 45 & 21 & $42,9 \%$ \\
\hline
\end{tabular}

Sur les 33 questionnaires remplis, seules quatre écoles ont complété le sondage de façon indépendante, sans aucun contact de la part de l'université Laurentienne.

Sur les 73 écoles contactées par l'Université Laurentienne, 24 ont complété le sondage, 5 ont refusé l'invitation et 44 n'ont pas répondu.

Sur les 24 écoles qui ont rempli le questionnaire du sondage, 17 ont répondu à l'invitation après un seul courriel, quatre ont répondu après avoir reçu un courriel de suivi et trois ont dû recevoir trois courriels. 
Sur les 44 écoles qui n'ont pas répondu au sondage, celles qui ont fourni leur adresse de courriel ont reçu jusqu'à trois courriels de rappel au sujet de l'état d'achèvement du sondage. Après ces trois courriels de suivi, ces écoles n’ont pas été contactées à nouveau.

Le Vision Institute of Canada a envoyé des copies imprimées de la lettre d'information et du questionnaire du sondage aux 18 écoles qu'il a été impossible de joindre par téléphone ou par courriel. Cinq de ces 18 écoles ont répondu par la suite.

Les réponses au sondage ont révélé qu'environ $25 \%$ des élèves portaient des lunettes (tableau 2). Cela suggère que, dans l'ensemble, il y a très peu de cas d'erreur de réfraction non corrigée. Toutefois, en raison de l'importance de la situation géographique sur l'accès aux services, nous avons comparé les taux de correction de la vision dans les collectivités éloignées à ceux des collectivités accessibles par la route. Les résultats sont présentés au tableau 3, qui compare également les taux dans les collectivités qui ont un optométriste visiteur à ceux d'autres collectivités.

\section{DISCUSSION}

Le tableau 1 montre que 32 écoles élémentaires des Premières nations de l’Ontario sont situées dans des collectivités éloignées et que les 45 autres sont situées dans des collectivités rurales, urbaines ou périurbaines. Les collectivités qui sont reliées uniquement par transport aérien et celles qui ne sont accessibles que par les routes de l'air ou de glace étaient considérées comme éloignées. Sur les 32 écoles des collectivités éloignées, 12 (37,5\%) ont répondu au sondage. Sur les 45 écoles des collectivités non éloignées, 21 (46,7 \%) ont répondu au sondage. Ces données portent à croire que les élèves vivant dans une collectivité éloignée des Premières nations pourraient avoir un meilleur accès aux services de soins de la vue. Le taux de réponse moins élevé observé pour les collectivités éloignées peut indiquer que l'incitation à répondre au sondage était moins forte que dans le cas des collectivités non éloignées. Cela peut être attribuable au fait que l'affectation de fonds aux Premières nations est influencée par des facteurs géographiques comme la distance du centre de service le plus proche et que, par conséquent, les collectivités isolées reçoivent plus d'attention que les collectivités non isolées ${ }^{19}$. Cependant, il arrive que les collectivités non éloignées des Premières nations se trouvent à plusieurs kilomètres d'un centre de service dispensant des soins complets de la vue. Les collectivités non éloignées ont indiqué que l'emplacement et les frais de déplacement étaient les principaux obstacles à l'obtention de services de soins de la vue complets. La distance moyenne entre les 33 communautés qui ont participé au sondage et l'optométriste le plus proche était de $196 \mathrm{~km}$.

Figure 1 : Taux de réponse au sondage

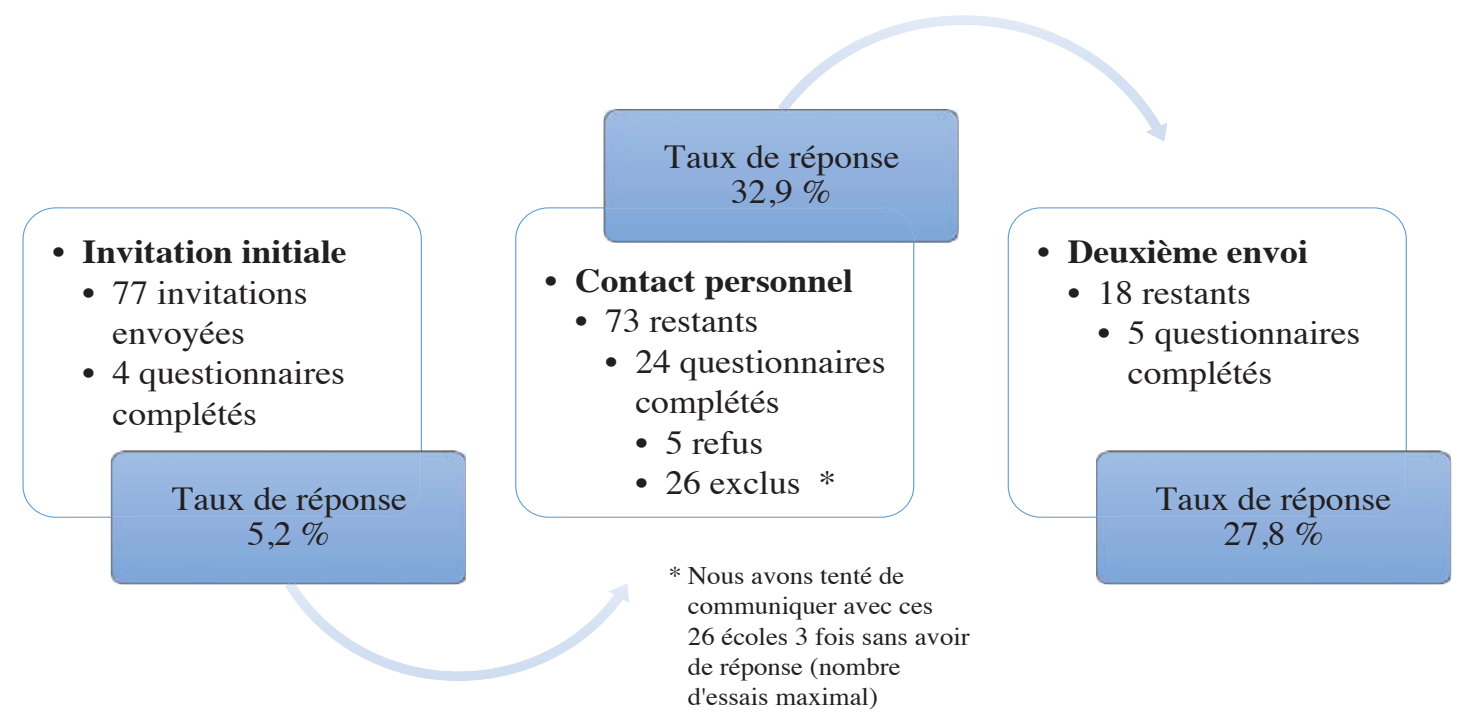


Tableau 2 : Pourcentage des enfants qui portent des lunettes dans chacune des écoles primaires

\begin{tabular}{|c|c|c|c|c|}
\hline $\begin{array}{l}\text { Communauté des Premières nations } \\
\text { (0) indique qu'un optométriste visite la communauté }\end{array}$ & $\begin{array}{l}\text { Nombre } \\
\text { d'élèves }\end{array}$ & $\begin{array}{l}\text { Nombre } \\
\text { avec des } \\
\text { lunettes }\end{array}$ & $\begin{array}{c}\text { Nombre } \\
\text { sans } \\
\text { lunettes }\end{array}$ & $\begin{array}{l}\text { Pourcentage } \\
\text { portant des } \\
\text { lunettes (\%) }\end{array}$ \\
\hline Première nation d'Aamjiwnaang & 11 & 0 & 11 & 0 \\
\hline Première nation d'Aroland & 91 & 21 & 53 & 28 \\
\hline Première nation Chippewas de Georgina Island & 15 & 2 & 13 & 13 \\
\hline Première nation Chippewas de Nawash & 71 & 14 & 53 & 21 \\
\hline Première nation Chippewas de Rama & 160 & 16 & 130 & 11 \\
\hline Première nation de Curve Lake & 53 & 9 & 44 & 17 \\
\hline Première nation de Deer Lake & 256 & 88 & 168 & 34 \\
\hline Première nation d'Eagle Lake & 27 & 3 & 24 & 11 \\
\hline Première nation de Fort Albany & 194 & 44 & 30 & $59(?) *$ \\
\hline Première nation de Fort Severn & 85 & 33 & 50 & 40 \\
\hline Première nation de Grassy Narrows & 187 & 30 & 157 & 16 \\
\hline Première nation de Keewaywin & 103 & 8 & 95 & 8 \\
\hline Première nation de Long Lake $\mathrm{n}^{\circ} 58$ & 97 & 4 & 7 & $37(?)$ \\
\hline Première nation Mattagami & 31 & 10 & 21 & 32 \\
\hline Première nation des Mississaugas de New Credit & 126 & 20 & 105 & 16 \\
\hline Première nation des Mohawks d'Akwesasne & 297 & 52 & 189 & 22 \\
\hline Première nation crie de Moose & 36 & 17 & 19 & 47 \\
\hline Première nation de Neskantaga & 49 & 12 & 37 & 24 \\
\hline Première nation de Nibinamik & 84 & 8 & 52 & 13 \\
\hline Première nation de North Caribou Lake & 148 & 18 & 130 & 11 \\
\hline Première nation de North Spirit Lake & 78 & 26 & 52 & 33 \\
\hline Première nation Northwest Angle $\mathrm{n}^{\circ} 37$ & 6 & 3 & 3 & 50 \\
\hline Première nation des Ojibways d'Onigaming & 46 & 16 & 30 & 35 \\
\hline Première nation de Pikangikum & 750 & 250 & 400 & $38(?)$ \\
\hline Première nation de Sagamok & 178 & 21 & 158 & 12 \\
\hline Première nation Shawanaga & 23 & 5 & 18 & 22 \\
\hline Première nation de Sheshegwaning & 10 & 2 & 8 & 20 \\
\hline Première nation de Shoal Lake $n^{\circ} 40$ & 33 & 7 & 26 & 21 \\
\hline Première nation Temagami & 33 & 7 & 24 & 23 \\
\hline Première nation Wabaseemoong & 300 & 52 & 200 & 21 \\
\hline Première nation de Wabigoon Lake & 12 & 3 & 9 & 25 \\
\hline Première nation Webequie & 144 & 18 & 126 & 13 \\
\hline Première nation de Weenusk & 28 & 7 & 21 & 25 \\
\hline TOTAL & 3762 & 826 & 2463 & Moy $=25,3 \%$ \\
\hline
\end{tabular}

*NOTA : (?) Indique des données qui peuvent refléter des rapports inexacts mais qui sont incluses dans les résultats finaux. 
Tableau 3: Pourcentage d'élèves qui portent des lunettes selon des groupes précis

\begin{tabular}{|c|c|c|}
\hline & $\begin{array}{l}\text { Pourcentage d'élèves portant } \\
\text { des lunettes }\end{array}$ & $\begin{array}{l}\text { Pourcentage d'élèves comptés } \\
\text { (par rapport à l'effectif total) }\end{array}$ \\
\hline \multicolumn{3}{|c|}{$\begin{array}{l}\text { La collectivité est-elle accessible uniquement par avion (non accessible par route } \\
\text { pendant toute l'année)? }\end{array}$} \\
\hline $\begin{array}{l}\text { Oui } \\
\text { Non }\end{array}$ & $\begin{array}{l}34 \% \\
20 \%\end{array}$ & $\begin{array}{l}86,4 \% \\
86,7 \%\end{array}$ \\
\hline \multicolumn{3}{|c|}{ Les familles visitent-elles un optométriste à l'extérieur de la collectivité? } \\
\hline $\begin{array}{l}\text { Oui } \\
\text { Non }\end{array}$ & $\begin{array}{l}29 \% \\
24 \%\end{array}$ & $\begin{array}{l}87,1 \% \\
84,4 \%\end{array}$ \\
\hline \multicolumn{3}{|c|}{ Y a-t-il d'autres types d'évaluations de la vision? } \\
\hline $\begin{array}{l}\text { Oui } \\
\text { Non }\end{array}$ & $\begin{array}{l}25 \% \\
25 \%\end{array}$ & $\begin{array}{l}78,9 \% \\
90,7 \%\end{array}$ \\
\hline \multicolumn{3}{|c|}{ Est-ce qu'un optométriste visite la collectivité? } \\
\hline $\begin{array}{l}\text { Oui } \\
\text { Non }\end{array}$ & $\begin{array}{l}29 \% \\
19 \%\end{array}$ & $\begin{array}{l}86,5 \% \\
91,8 \%\end{array}$ \\
\hline
\end{tabular}

Notre objectif principal était de comparer les taux de correction de la vue aux normes attendues, ce qui pourrait laisser entrevoir un besoin de services. Les résultats regroupés présentés au tableau 2 indiquent que le pourcentage d'enfants portant des lunettes dans l'ensemble des 33 écoles ayant répondu était de $25,3 \%$, ce qui est très semblable au taux normal prévu dans la population générale ${ }^{8}$. Toutefois, d'après les résultats des deux écoles mentionnées dans l'introduction, cette valeur devrait être considérablement plus faible (environ 4,4\%). Puisque cette étude a utilisé des données autodéclarées pour recueillir de l'information, certaines formes de biais peuvent expliquer pourquoi ce résultat n'est pas vrai-

ment représentatif de la population. Par exemple, les données ont été fournies par les représentants de l'école, ce qui augmente le risque d'un biais d'acceptabilité sociale. Il est possible que les répondants au sondage aient fourni, de façon non intentionnelle, des données qui dépeignent leur école élémentaire ou la collectivité des Premières nations de façon plus favorable. Ajoutons que les représentants de l'école ayant également été choisis par le directeur de l'école et ayant peutêtre reçu leurs directives de deuxième ou de troisième main, ils n'avaient peut-être pas une compréhension concrète du but de l'étude ou de l'importance d'une collecte précise des données. Cela peut avoir affecté la validité globale de l'étude et les résultats peuvent ne pas être généralisables à l'ensemble de la population.

Lorsque nous avons exploré les taux de correction de la vue à travers les collectivités, nous avons reconnu que certaines régions avaient un plus grand besoin de services, basé sur un pourcentage plus faible d'élèves portant des verres correcteurs. Selon les résultats du tableau 3, 34\% des élèves qui fréquentaient une école élémentaire située dans une collectivité isolée ou accessible seulement par transport aérien portaient des lunettes, ce qui est supérieur à la valeur moyenne prévue de $25 \%^{8}$. D'autre part, $20 \%$ des élèves qui fréquentaient une école non isolée administrée par la bande portaient des lunettes, ce qui est inférieur à la moyenne prévue. Ces résultats suggèrent que les collectivités des Premières nations qui ne sont accessibles que par la voie des airs (ou isolées) sont plus susceptibles de recevoir suffisamment de services de soins de la vue que les collectivités non éloignées. Les données du tableau 3 portent également à croire que le pourcentage d'élèves qui portent des lunettes est plus élevé dans les collectivités des Premières nations dont les habitants sont en mesure de visiter un optométriste à l'extérieur de la collectivité que dans celles qui n'ont pas cette possibilité. Cependant, dans les familles qui ne visitent pas un optométriste, $24 \%$ des enfants portent des lunettes, ce qui est encore très proche de la moyenne attendue.

Dans l'ensemble, $25 \%$ des élèves portaient des lunettes, indépendamment de toute autre forme d'évaluation de la vue (par exemple, un simple test de dépistage effectué par un enseignant ou une infirmière d'école), ce qui est conforme à la norme attendue. Enfin, le pourcentage d'élèves qui portaient des lunettes (29\%) dans les collectivités qui ont un optométriste visiteur était supérieur au pourcentage d'élèves qui portaient des lunettes (19\%) dans les collectivités qui n'avaient pas accès à un optométriste visiteur. Cela porte à croire que le modèle de l’optométriste visiteur est très efficace.

Le tableau 3 montre que, de façon générale, les pourcentages d'enfants portant des lunettes sont relativement élevés dans chaque catégorie. Cependant, aucun des groupes ne représente $100 \%$ de la population des élèves. Les échantillons des 33 écoles (représentant $43 \%$ de la population cible) ne représentaient pas l'ensemble des élèves qui fréquentaient chaque école. Bon nombre des écoles participantes avaient des taux d'absence élevés le jour où les données ont été recueillies. Il est intéressant de spéculer que les enfants en bonne santé fréquentent la classe régulièrement et sont donc plus susceptibles d'avoir été inclus dans cette étude. Cela augmenterait le pourcentage global d'enfants portant des lunettes et conduit à un biais de sélection. L'échantillon statistique utilisé pour l'analyse (pop- 
ulation d'élèves en bonne santé) n'a pas été correctement randomisé et n'est donc pas représentatif de l'ensemble de la population. De plus, les 33 écoles examinées dans cette étude (3 762 élèves au total) représentaient moins de $39 \%$ de la population totale des élèves des 77 écoles primaires des Premières nations en Ontario (9 698).

\section{LIMITES}

Cette étude a été difficile à mener et nous reconnaissons que diverses limites méthodologiques doivent être prises en compte lors de l'interprétation des résultats.

Premièrement, les écoles ont été contactées indirectement, par l'entremise de deux organismes tiers, comme la lettre d'invitation a été envoyée par les Chiefs of Ontario et le suivi a été fourni par l'Université Laurentienne. Cette approche indirecte reposait sur notre partenariat avec les Chiefs of Ontario et nous nous sommes efforcés de faire en sorte que nos recherches soient pertinentes et respectueuses, mais elle a peut-être causé une certaine confusion chez les répondants. En outre, les invitations ont d'abord été envoyées aux représentants des conseils scolaires, qui ont ensuite transmis le matériel aux directeurs d'école respectifs. Cette méthode indirecte peut avoir eu comme résultat que certains participants potentiels n’ont pas reçu le questionnaire. Cette préoccupation a été confirmée par plusieurs écoles qui ont déclaré n'avoir jamais reçu le questionnaire, ce qui a probablement contribué à la petite taille de notre échantillon.

Deuxièmement, il était difficile de communiquer avec le directeur ou un représentant approprié par téléphone. Beaucoup voyagent ou sont très occupés avec les programmes scolaires. De plus, de nombreux secrétaires d'école n'étaient pas familiers avec l'étude et certains se sentaient mal à l'aise de fournir une adresse de courriel. Il y avait donc de nombreux obstacles à la réception du questionnaire du sondage.

Ces limites reflètent certaines des réalités de la réalisation de recherches auprès des Premières nations. En dépit de ces limites, nous croyons que nos résultats sont importants parce qu'ils représentent une avancée significative dans le niveau d'information disponible à ce jour et démontrent qu'il existe un besoin au niveau des collectivités, ce qui pourrait guider l'allocation de ressources limitées.

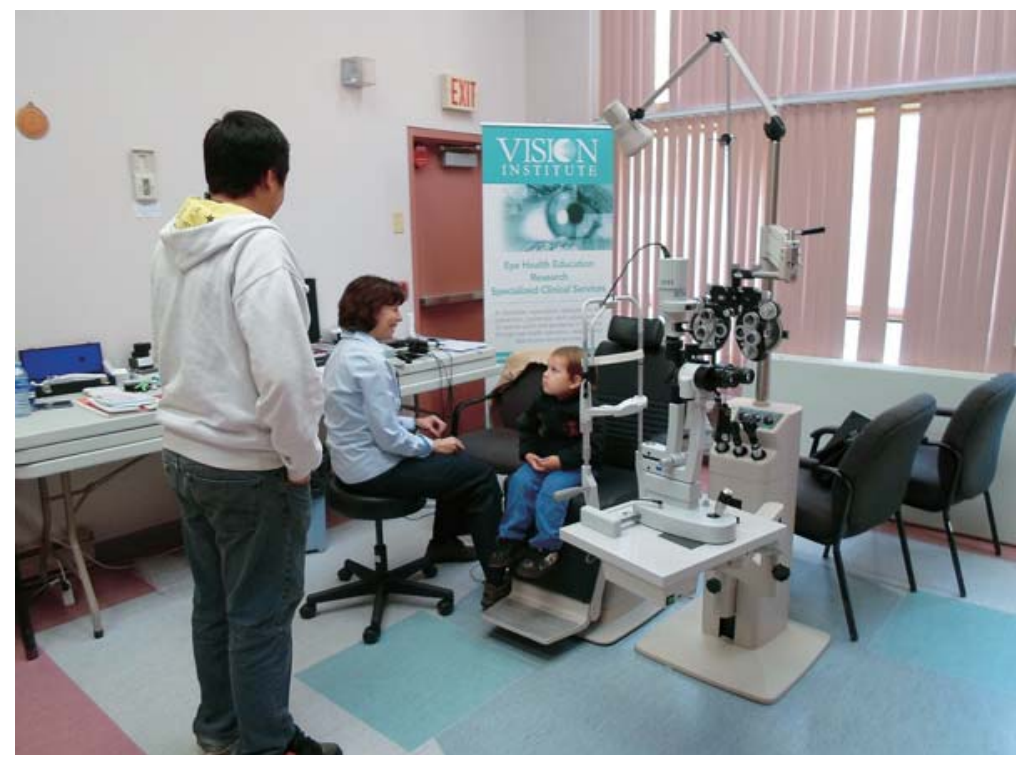

Dr Catherine Chiarelli a offert un examen oculaire complet à un jeune étudiant de la Première Nation de Sagamok.

RECOMMANDATIONS POUR LES ACTIVITÉS FUTURES DE RECHERCHE

Plusieurs pistes d'amélioration se dégagent de nos travaux. Une copie du questionnaire et de la lettre d'information doit être envoyée directement aux directeurs d'école. Bien que le suivi par téléphone prenne du temps, il est important, puisque cette méthode a atteint un taux de réponse de 32,9\%. L'envoi d'un deuxième courriel est également important et a permis d'atteindre un taux de réponse de $27,7 \%$ parmi les écoles qui n'avaient pas répondu à d'autres approches. 
En outre, les dirigeants de la collectivité devraient être informés des objectifs de l'étude et des procédures à l'avance. Cela pourrait améliorer la participation ou du moins encourager une évaluation de l'adéquation des services de soins de la vue disponibles dans la collectivité. En outre, certains directeurs d'école n'étaient peut-être pas à l'aise de prendre la décision de participer au projet de recherche sans en avoir tout d'abord parlé au conseil de bande et demandé son approbation.

Il peut également être avantageux qu'un membre du personnel des Chiefs of Ontario effectue le suivi auprès de chaque Première nation, puisqu'ils ont déjà un lien avec ces collectivités. En procédant de cette façon, les participants pourraient se sentir plus à l'aise pour poser des questions, discuter de leurs préoccupations ou demander des renseignements supplémentaires, ce qui pourrait conduire à une décision plus éclairée et au désir de participer à l'étude.

CONCLUSIONS

Dans l'ensemble, cette étude visait à évaluer, à titre préliminaire, le niveau et la qualité des services de soins de la vue offerts aux écoles primaires des Premières nations de l'Ontario. La principale statistique d'intérêt était le nombre d'enfants portant des lunettes, puisque la comparaison avec une norme attendue est le moyen le plus facile à grande échelle d'inférer indirectement le niveau d'erreur de réfraction non corrigée dans chaque école. Dans cette étude, qui avait un taux de réponse global de $43 \%, 25 \%$ des enfants fréquentant les écoles primaires des Premières nations portaient des lunettes, ce qui est très proche de la norme attendue. La cohorte des collectivités qui ne sont accessibles que par transport aérien avait le pourcentage d'enfants portant des lunettes le plus élevé, tandis que les collectivités qui n'avaient pas d'optométriste visiteur avaient le pourcentage le plus faible. Cependant, ces résultats ne sont pas conformes à ceux d'études comparables menées sur une base individuelle au niveau des collectivités des Premières nations, et il faut donc veiller à interpréter les présents résultats avec prudence. Un biais d'acceptabilité sociale peut expliquer le nombre plus élevé d'élèves qui portent des lunettes dans les écoles où des études antérieures ont montré que le pourcentage d'élèves portant des lunettes était beaucoup plus bas que la valeur prévue. Étant donné que cette valeur est beaucoup plus faible dans trois écoles (voir les annexes 1 et 2), ce biais peut avoir influencé plusieurs réponses des participants. De plus, un biais de sélection est probablement attribuable à la faible fréquentation de certaines écoles, de sorte qu'un grand nombre d'enfants - sans doute issus de familles défavorisées sur le plan économique ou faiblement scolarisées- n'ont pas été comptés. Par conséquent, un mode de distribution des questionnaires de sondage amélioré et plus responsable, dans lequel ils sont envoyés directement aux dirigeants des Premières nations, pourrait permettre d'améliorer la validité de ce type d'étude.

RECOMMANDATIONS POUR AMÉLIORER LES SERVICES DE SOINS DE LA VUE OFFERTS AUX ÉCOLES PRIMAIRES DES PREMIËRES NATIONS

1. Chaque école primaire des collectivités des Premières nations doit recevoir la visite d'un optométriste au début de chaque année scolaire afin de fournir des examens de la vue complets et des lunettes à la population étudiante.

2. Chaque enfant qui a besoin de lunettes doit en recevoir deux paires pour mieux se conformer au port de lunettes (voir l'annexe 1).

3. Les enseignants et le personnel de l'école devraient être plus impliqués et savoir quels sont les enfants qui portent des lunettes afin d'encourager et de soutenir la conformité avec le port de lunettes.

4. Chaque école primaire des Premières nations profiterait de la création d'un comité de la vue des enfants composé de deux ou trois membres du personnel de l'école qui pourraient travailler pour éduquer les enseignants et les parents sur la vue des enfants et aider à identifier les élèves qui ont besoin de soins urgents.

\section{REMERCIEMENTS}

Les auteurs tiennent à remercier l'Association canadienne des optométristes d'avoir offert une subvention éducative sans restriction à l'appui de ce projet. Ils sont également reconnaissants à Mme Donna Atkinson du Centre de collaboration nationale pour la santé autochtone pour la relecture de ce document, Mme Bernadette deGonzaque, de l'Organisation des Chiefs of Ontario, et M. Tony Jocko, de l’Union des Indiens de l'Ontario. • 


\section{RÉFÉRENCES}

1. Statistique Canada Les peuples autochtones au Canada : Premières Nations, Métis et Inuits : Enquête nationale auprès des ménages (2011) Ottawa (Ontario) : Gouvernement du Canada, 2013.

2. Macdonald D., Wilson D. Honteuse négligence : la pauvreté chez les enfants autochtones au Canada; Centre canadien de politiques alternatives; mai 2016; ISBN 978-1-77125-284-3.

3. Greenwood M, de Leeuw S, Lindsay NM, et coll., éd. Determinants of Indigenous Peoples' Health in Canada: Beyond the Social; Toronto, Canadian Scholars' Press; août 2015; ISBN: 9781551307329 (en anglais).

4 (en anglais). King M. Chronic diseases and mortality in Canadian Aboriginal peoples: Learning from the knowledge. Chronic Dis Can 2010; 31:2-3.

5. Smylie J. The health of Aboriginal peoples. Dans : Raphael, D., éd Social Determinants of Health: Canadian Perspectives, 2de éd. Toronto: Canadian Scholars Press Inc., 2009.

6. Smylie J, Adomako P., éditeurs. Indigenous Children's Health Report: Health Assessment in Action. Toronto (Ontario) : Centre for Research on Inner City Health, 2009.

7. Harvey EM, Dobson V, Miller JM. Prevalence of high astigmatism, eyeglass wear, and poor visual acuity among Native American grade school children. Optom Vis Sci 2006 Apr;83(4):206-12.

8. Chiarelli C, Chris AP. État de la vue des enfants des Premières Nations : le Projet sur les soins oculovisuels dans la Première Nation Sagamok Journal canadien d'ophtalmologie 2013;75(4):6-10 (en anglais)

9. Chua V, Mitchell P. Consequences of amblyopia on education, occupation, and long term vision loss. Br J Ophthalmol 2004;88(1):1119-21.

10. Castanes MS. Major review: The underutilization of vision screening (for amblyopia, optical anomalies and strabismus) among preschool age children. Binocul Vis Strabismus Q 2003; 18(4): 217-32.
11. Tobin, P. Preschool vision screening for Aboriginal children in British Columbia: A needs assessment (en anglais). Prince George, BC. Rapport préparé pour le CCNSA, 2007.

12. Atkinson DL Preschool Vision Screening and Aboriginal Eye Health : An Aboriginal Eye Health and Literature Review. NCCAH, avril 2007.

13. Rasmussen C, Sherman J, Baydala L. Learning patterns and education of Aboriginal children: A review of the literature. Can J Native Studies 2004;2:317-42.

14. Ganz M, Xuan Z, Hunter, D. Prevalence and correlates of children's diagnosed eye and vision conditions. Ophthalmology 2006; 113(12):2298-306.

15. Williams C, Northstone K. Howard M, et coll. Prevalence and risk factors for common vision problems in children: Data from the ALSPAC study. Br J Ophthalmol 2006; 92(7):959-64.

16. Majeed M, Williams C, Northstone K, et coll. Are there inequities in the utilization of childhood eye-care services in relation to socio-economic status? Evidence from the ALSPAC cohort. $\mathrm{Br} J$ Ophthalmol 2008; 92(7):965-9.

17. Schlenther G; Tahhan N, Stretton S, et coll. Aboriginal eyecare: A review of services in NSW [online] Aboriginal \&t Islander Health Worker Journal, 2006; 30(4): 7-8.

18. Harris PA, Taylor R, Thielke R, et coll. Research electronic data capture (REDCap): A metadata-driven methodology and workflow process for providing translational research informatics support. $J$ Biomed Inform 2009 Apr; 42(2):377-81

19. Joint AFN/INAC BOFF Working Group Final Report: A Study of Educational Costs Drivers to First Nations Education. Simon Management Services; April 2006

Les annexes 1-4 peuvent être consultées en ligne à www.opto.ca

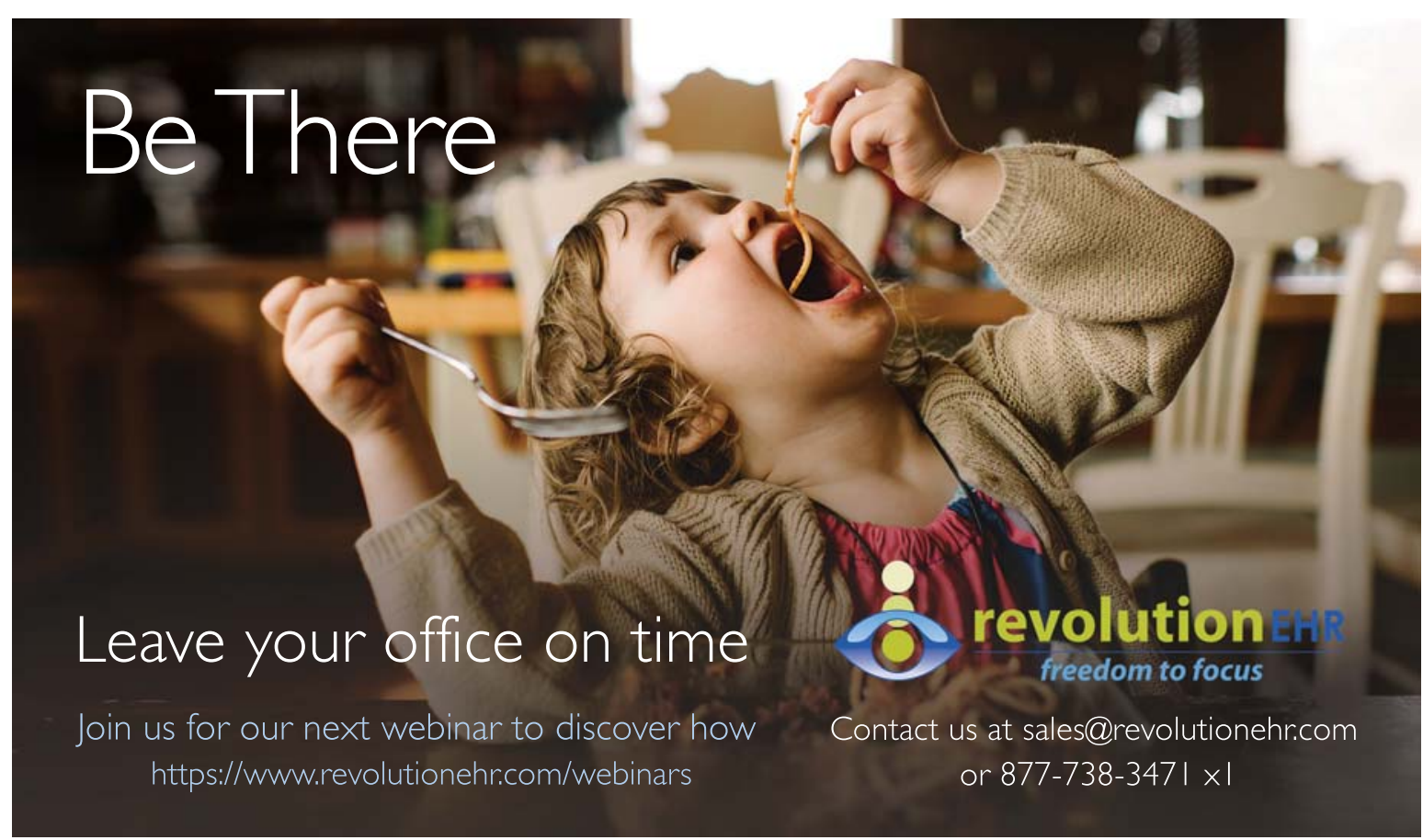

